\title{
Case Report Of Adenocarcinoma On Palpebral Of Rotweiler Dog: A Histopathological Evaluation
}

\author{
Dwinna Aliza ${ }^{1,{ }^{*},}$ Nazaruddin Nazaruddin ${ }^{1}$, Ari R. Siregar ${ }^{2}$, Arman Sayuti ${ }^{3}$, Etriwati Etriwati ${ }^{1}$, Denny I. Hasan ${ }^{1}$, \\ and Awaluddin Awaluddin ${ }^{1}$ \\ ${ }^{1}$ Laboratory of Pathology, Veterinary Medicine Faculty, Universitas Syiah Kuala \\ ${ }^{2}$ Veterinary Practitioner, Medan. \\ ${ }^{3}$ Laboratory of Clinic, Veterinary Medicine Faculty, Universitas Syiah Kuala.
}

\begin{abstract}
Eyelid neoplasms cases are the most frequently recorded ophthalmic tumors in dogs, which mostly benign and only few are reported as being malignant. Adenocarcinoma is one of malignant tumor reported in dog eyes beside fibrosarcoma, squamous cell carcinoma, and hemangiosarcoma. A case of adenocarcinoma in palpebral of a 6-year-old male rotweiller dog with 6- month history of a progressive mass on the left eye was presented. Pathological examination showed a severe exophytic irregular pink to red cauliflower-like mass occupying all surface of the eye lid. A transpalpebral enucleation was performed to collect the mass, followed by histopathological slide preparation using Hematoxylin-Eosin (HE) staining. Histopathological examination showed various sizes lobules separated by connective tissue. Many angioneogenesis figure were also observed. The irregular arranged of anaplastic cells was obviously seen with hyperchromatic and pleomorphic of the nuclei and various shapes and sizes of cell structures as well. There was no sign of encapsulated area, however, few mitotic figures were seen. The histopathological examination result confirmed the presence of adenocarcinoma.
\end{abstract}

Keywords: adenocarcinoma, histopathology, dog's palpebral, anaplastic cell.

\section{Introduction}

Dogs are susceptible to tumor and carcinoma cases, which is proven by numerous reports from research center around the world. The two-decade demographic study of the Veterinary Medical Database to more than 74,000 dogs in America, found that neoplastic disease was the most common terminal pathological process in 73 of 82 canine breeds and the most common cause of death in dogs over 1 year of age [1]. Other reports in Northwestern of Italy held by Piedmont Canine Cancer Registry from 2001-2008 showed that 1175 cases was confirmed tumours cases by histopathological diagnosis, which the incidence rate was 804 per 100,000 dog per year for malignant tumours and 897 per 100,000 dog per year for benign tumours [2]. In addition, a total of 2,546 of 130,684 insured dogs were diagnosed tumours in The United Kingdom [3], 381-1126/100,000 dogs in Bulgaria (4), and 1568 cases were diagnosed in Croatia from 2006-2009 [5].

Eyelid tumors are commonly found in dogs which grouped as ophthalmic neoplasm. It presented as raised cutaneous masses that may have originated from epithelial, mesenchymal or melanogenic cells [6]. Sebaceous gland tumors, squamous cell papillomas, and melanocytic tumors are frequently found in the eyelids of dogs and tend to be benign: although their progressive growth can be associated of self-trauma and chronic irritation. Sebaceous gland tumors, generally arise from tarsal glands [7]. Among these, sebaceous adenomas, sebaceous epitheliomas, and sebaceous adenocarcinomas, are commonly encountered.

Dog eyelid consist of anterior and posterior part, which is folds of tissue lined by skin anteriorly and palpebral conjunctiva posteriorly [8]. Most tumor affecting canine eyelids are tumors generally encountered in the skin 9]. They include melanocytic tumors, sebaceous gland adenomas, histiocytic and mast cell tumors, squamous papillomas and carcinomas, trichoblastomas and trichoepitheliomas [6]. A total of $64.3 \%$ of the eyelid neoplasms corresponded to meibomian gland tumors. Epithelioma (22.4\%) and adenoma $(15.4 \%)$ of the meibomian glands were the most frequent ophthalmic tumors in dogs [7].

Adenocarcinoma is a malignant tumor of glandular epithelium with a gross appearance varies with its location, the gland tissue of which it consists, and the age of the growth. Although adenocarcinoma commonly reported in dogs $(10 \%-18 \%)$ are in colon, kidney, rectum, mammary and lymphoma [4], however this study case present the adenocarcinoma of eyelid.

\footnotetext{
Corresponding author: dwinna.aliza@unsyiah.ac.id
} 


\section{Materials and Methods}

A transpalpebral enucleation was performed to collect a severe exophytic cauliflower-like mass on the top surface of left eye lid of a 6-year-old male rotweiller dog. Subsequently, the mass was fixed in $10 \%$ neutral buffered formalin, embedded in paraffin, sectioned to 5 $\mu \mathrm{m}$ thickness, and stained with Hematoxylin-Eosin (H\&E). The slide was then observed using light microscope and documented by photomicroscope (Olympus)

\section{Results and Discussion}

The anatomical pathology examination on the rotweiller dog showed a progressive exophytic pink to reddish cauliflower mass covering all surface of left eyelid (Figure 1). This mass history was initially from a $5 \mathrm{~mm}$ nodule which develop progressively within 6 months become approximately $7 \mathrm{~cm}$ in diameter, thus the dog could not open the eye. Furthermore alopecic was observed on the mass and expanded to surrounding ophthalmic area. Previous study by [10] reported the morphology of adenocarcinoma mass on terrier breed dog that on mucous surfaces it may simply be a welldefined, rather flat, undulating, thickened area which has the characteristics of chronic focal inflammation. At the other it may be much more conspicuous and take the form of a head of cauliflower. Furthermore [11] added that the adenosarcoma gross appearance display a pinklike appearance and are stationary.
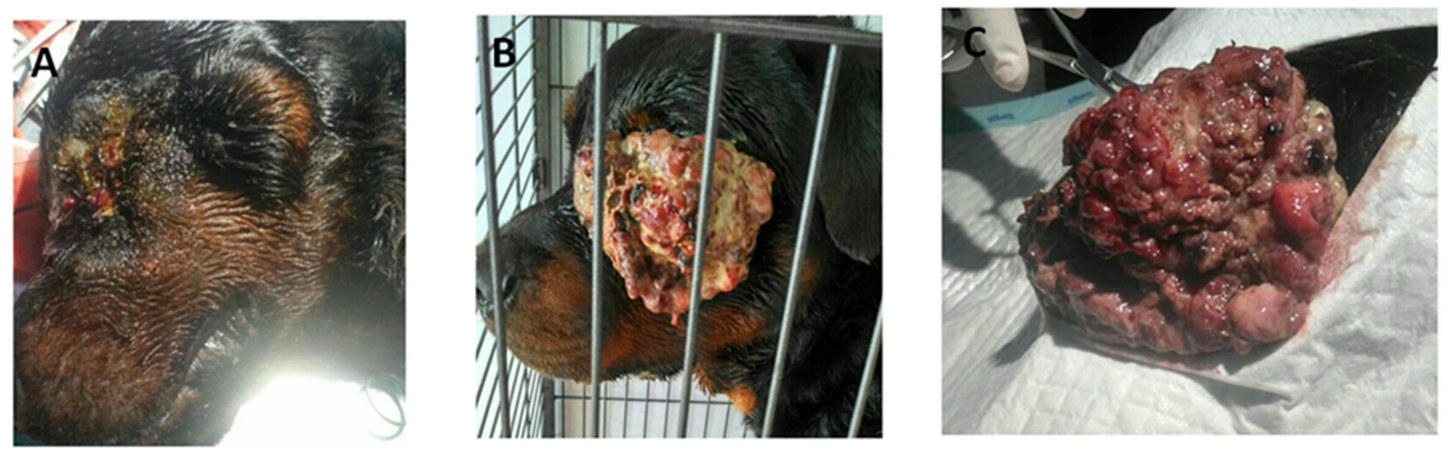

Figure 1. The anatomical pathology demonstrates an exophytic, irregular reddish-pink mass on the surface of left palpebral of 6year-old rotweiller $\operatorname{dog}(\mathrm{A}$ and $\mathrm{B})$. Transpalpebral enucleation of the progressive tumor (C).

Histopathological examination showed various sizes lobules separated by connective tissue which arrange loosely with several angioneogenesis figure (Fig. 2B). The irregular arranged of anaplastic cells was obviously seen with hyperchromatic and pleomorphic of the nuclei and various shapes and sizes of cell structures as well (Fig. 2C). There was no sign of encapsulated area, however, few mitotic figures were seen (Fig. 2A,D). Microscopically, several signs indicated carcinoma cases such as enlargement of the nucleus is an indication of rapid cell growth and an embryonic cell type, multiple nuclei in a cell which indication of a rapidly growing embryonic cell type. Furthermore, the enlargement of the nucleolus is associated with rapid cell growth $[4,7]$.

A report by [12] stated that neoplastic cells of adenocarcinoma were round to polygonal, pleomorphic with abundant cytoplasm, with irregularly shaped immature nuclei characterized by a high grade anisokaryosis and multiple nucleoli. Additionally [8] also reported that the cells of adenocarcinoma were basophilic, columnar and cuboidal with basal, prominent, hyperchromatic, condensed nuclei and moderate amount of cytoplasm. There were necrosis, inflammatory cell infiltration, and severe desmoplasia in association with neoplasms that infiltrated the tissue of the organ.
Hyperchromasia of the cell is commonly seen in some carcinoma tissues. The more embryonic the cell the more intensely the cell stains with hematoxylin, especially the nucleus [13]. The intensity of the hematoxylin of the cell compared with the normal adult cell gives an indication as to the maturity of the cell. The embryonic cells growth are no longer under the control of growth regulating and maturating factors $[7,15]$. The increased number of mitotic figures indicated malignant type of tumor. The more rapidly the cells are multiplying the greater is the chance the mitotic figures will be found. When mitotic figures are abundant it shows the level or grade of the carcinoma [14].

The adenosarcoma tissue, which effaced the normal glandular tissue, was composed of a uniform population of epithelial cells that had two morphological appearances. The first appearance consisted in acini and tubules. The lumen of glandular structures contained an eosinophilic secretory material. Epithelial cells had moderate amounts of cytoplasm and round, vesicular nuclei. The second appearance consisted of cells that were small, arranged in nests, and had scant cytoplasm, ovoidal nuclei, and dense chromatin. Mitotic figures (up to 10 per high-power field) were numerous. The surrounding connective tissue was arranged loosely and had a mucinous-to cartilaginous appearance $[15,16]$. 

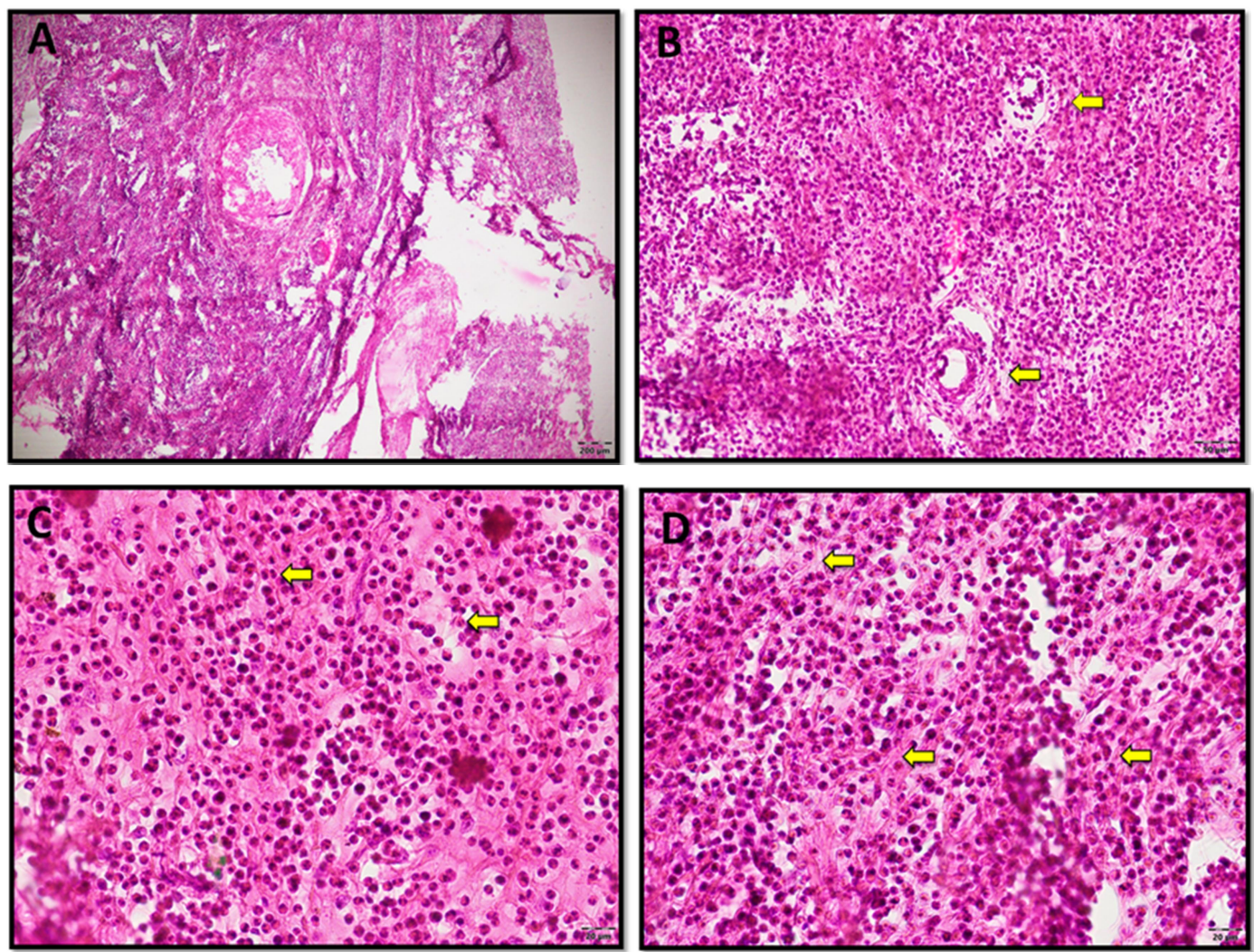

Figure 2. The histopathological figure of adenocarcinoma on left eyelid of rotweiller dogs. (A) No sign of encapsulated area, HE 10X, (B) angioneogenesis, HE 10X, (C) anaplastic cells showed enlargement and hyperchrome of the nucleolus, various sizes lobules separated by connective tissue (arrow) HE 40X, (D) mitotic demonstrated as two nucleoli in a cell, HE 40X.

Several purebreds of dog are known to have a high incidence and elevated risk of specific cancer subtypes, sometimes even more than one subtype. Such breed specific risk reflects the underlying genetics of the different breeds. A few breeds of dogs who always has carcinoma cases were Bernese Mountain Dog (BMD), flat-coated retriever, rottweiler and golden retriever. Previous research found that carcinoma cases in rottweiler dog was osteosarcoma and malignant histiocytosis (HS) $[17,18]$.

Since many of the key phenotypes are characteristics of the particular breed, their presence in the breed had been positively selected, resulting in high frequency of the genes that cause these specific phenotypes [17]. While some diseases have simple inheritance patterns, others, including cancers, are likely to be more complex. The genetic background of some purebred dogs may predispose the breed to a higher risk for specific cancers, or cancers in general. This increased risk of cancer in purebred dogs that may be leveraged to accelerate the process of cancer gene discovery from a comparative perspective $[17,18]$.

Some DNA (herpes viruses, papova and adenovirus) and RNA viruses (retroviruses) are named tumour viruses as they are proved to cause cancer in infected cells. Several exogenous factor such as chemical, food, and radiation lead to DNA damage and produce cell alterations. However, hormones are another endogenous factor caused specific cancer type such gland tumour including adenosarcoma [19]. Moreover, that cancer is more commonly observed in older dog which supports the concept of over the course of time, a combination of factors leads to a normal cell's transformation into a cancer cell $[18,19]$.

\section{Conclusion}

The histopathological examination results confirmed the presence of adenocarcinoma by the occurrence of various sizes lobules of anaplastic cells.

\section{References}

1. Biller B, Berg J, Garrett L: 2016 AAHA Oncology Guidelines for Dogs and Cats: Veterinary Practice Guidelines. J Am Anim Hosp Assoc. 2016;52:181-204.

2. Baioni E, Scanziani E: Estimating canine cancer incidence: findings from a population-based tumour registry in northwestern Italy. BMC Vet Res. 2017; 13:203-219.

3. Dobson J, Samuel S, Milstein H, et al.: Canine neoplasia in the UK:Estimates of incidence rates from a population of insured dogs. $J$ Small Anim Pract. 2002; 43(6):240-246.

4. Todorova I: Prevalence and etiology of the most common malignant tumours in dogs and cats. Bulgarian J Vet Med. 2006; 9(2):85-98. 
5. Zuckermann ICS, Severin K, Hohsteter M, et al.: Incidence and types of canine tumours in Croatia. Veterinarski Arhiv. 2013; 83(1):31-45.

6. Willis AM, Wilkie D: Ocular oncology. Clinical techniques in small animal practice. 2001;16(1):77-85.

7. Komaromy AM., Ramsey DT, Render JA, Clark P: Primary adenocarcinoma of the Gland of the nictitating membrane in a cat. $J$ American Anim Hospital Assoc. 1997; 33:333-336.

8. Patnaik AK, Hurvitz AI, Johnson GF: Canine Intestinal Adenocarcinoma and Carcinoid. Vet. Pathol, 1980;17:149-163.

9. Schiffman JD, Breen M: Comparative oncology: what dogs and other species can teach us about humans with cancer. Phil. Trans. R. Soc. B. 2015; 370:20140231.

10. Tavasoli A, Javanbakht J, Shafiee R, et al.: Citological and histopathology features of meibomian adenocarcinoma in a dog terrier breed. $J$ clin exp Pathol. 2012; 2-5.

11. Gopal K, Vairamuthu S, Balachandran C, et al. : Meibomian Gland Carcinoma in a Labrador Dog. Indian Vet. J. 2018; 95(01):81-82.

12. Barsotti G, Ressel L, Finotello R, et al.: Primary corneal squamous cell carcinoma in a Dog: Clinical and histopathological evaluation. Case Reports Vet Med. 2012;3-6.
13. Demirutku A, Devecioglu Y, Eravci E, et al.: Meibomian adenoma in a Syrian hamster (Mesocricetus auratus): a case report. Veterinarni Medicina. 2013; 58(12):641-644.

14. Nordio L, Fattori S, Giudice C: Fibrosarcoma of the eyelid in two sibling Czech wolfdogs. Open Vet $J$. 2017; 7(2):95-99..

15. Yuan DW, Goo MJ, Yang HJ, et al.: Adenocarcinoma of Meibomian Glands and Moll Glands in the Eyelid of Canine. J. Life Sci. 2008; 3:414-417.

16. Chandrasekaran D, Kavitha S, Thangapandyan M, et al.: Pulmonary Adenocarcinoma in A Dog-A Case Report. Indian Vet J. 2016; 93(04):58-59.

17. Gillard M, Cadieu E, De Brito C, et al.: Naturally occurring melanomas in dogs as models for non-UV pathways of human melanomas. Pigment cell melanoma Res. 2014; 27:90-102.

18. Dobson JM:Breed-predispositions to cancer in pedigree dogs. ISRN Vet Sci. 2013.

19. Todorova I: Prevalence and etiology of the most common malignant tumours in dogs and cats. $B J V M$. 2006; 9(2):85-98. 